\title{
Attendance System with Face Recognition
}

\author{
Tanya Sinha, Abhisekh Ghosh, G. Manju
}

\begin{abstract}
Marking Attendance is the most common way to know the physical presence of an individual. But it is challenging when it comes to manual attendance system, which is followed in most of the places. Calling out each student's registration number one by one is a tedious task. Day by Day the number of students in schools and universities is increasing hence, making it more difficult in managing and maintaining the attendance records. Automation is the need in every sector to reduce the human effort. Computer vision is a part of automation where computer replicates the human vision system and performs an understanding of useful information from images. It is a boon for many problems, attendance system can also be transformed from manual sheets to face recognition. This paper proposes a framework for developing an attendance system using Face Recognition. This system comprises an Android Application that can be installed on professor's mobile phone. Through the application, the camera can be unlocked to capture images. Each student's image is captured and stored for training. OpenCV is used with a machine learning algorithm to search for faces within a single image. Once faces are found it is trained using KNN (K Nearest Neighbor) classifier. New images are compared with pre-existing images stored in the database using the KNN algorithm. Attendance is automatically recorded when the faces are matched, if not either the student is new and it is added in database or it is declared as a false attendance i.e., proxy. In this way accuracy is also maintained, thus making attendance process easier and efficient.
\end{abstract}

Keywords : Android Application, Database, Face Detection, Face Recognition, Machine learning.

\section{INTRODUCTION}

Deep learning is one of the interesting areas of technical innovations these days. It allows the machinery to work by itself by resulting outputs for some given datasets as inputs with the help of various learning algorithms. At present attendance is appraised an important concern for an educational organization as most of the universities maintain an attendance sheet to capture student's punctuality. With the growth of deep learning, the machine automatically detects the attendance performance of the student and helps in the maintaining records. In general, attendance can be taken in two ways - Manual Attendance System (MAS) and Automated Attendance System (AAS). In MAS, the teacher

Revised Manuscript Received on April 04, 2020.

* Correspondence Author

Tanya Sinha*, Computer Science and Engineering, SRMIST Ktr Campus, Tamil Nadu. Email: sinha.tanya26@gmail.com

Abhisekh Ghosh, Computer Science and Engineering, SRMIST Ktr Campus, Tamil Nadu. Email: abhishekplanet3@gmail.com

G. Manju, Computer Science and Engineering, SRMIST Ktr Campus, Tamil Nadu. Email: manju.g@ktr.srmuniv.ac.in

(C) The Authors. Published by Blue Eyes Intelligence Engineering and Sciences Publication (BEIESP). This is an open access article under the CC BY-NC-ND license (http://creativecommons.org/licenses/by-nc-nd/4.0/) needs to pronounce the student's registration number or name separately and give the attendance accordingly but it's very tiring and time-consuming. This system is not accurate because the teacher may miss some names or get confused when students have similar names. The most common failure of the system is proxy issues and fake attendance where students give attendance on behalf of their friends. There are few students who never attend the class or bunk the class, but still, they manage in getting full attendance. Hence, the traditional attendance system is not authentic it is unreliable. To overcome these issues and making the attendance system more reliable and user-friendly we go with Automated Attendance System (AAS). Modern Attendance System (MAS) is an automated process analyzing the physical presence or absence of the students in the classroom by implementing learning algorithms. Face Recognition are of two types, first type is Two-Dimensional face recognition which has few difficulties including variations in facial expression, brightness and image resolutions. The other type is Three-dimensional face recognition which has shown its excellence in areas like illuminations and facial expressions but this cannot be implemented easily because of its complexity and computation cost implementing 3D in devices. In our research, we have used two-Dimensional Face Recognition. The whole paper is divided into serval sections. The next section describes previous works followed by section III describing methods adopted in the system. Next Section IV emphasizes the results and discussions, and the limitations of the system in Section V. Modern Attendance System can also find out the position and relative distance between the students and the camera. The camera will detect the appearance of students providing us a count on the number of students present in the classroom.

\section{STATE OF THE ART}

In the paper by [1], the ideology for the student's attendance system has been executed using Machine Learning algorithms. The student's face, as well as performance, is automatically detected using the system and feedback on various subjects like Mathematics, Science, etc is maintained. Hence, attendance can be marked by face recognition. On recognition, the attendance percentage and marks of students can be obtained. When we employ face detection as well as recognition algorithms, students face is automatically detected when someone enters the classroom and based on recognition attendance is updated. Numerous algorithms like Viola-Jones are utilized in distinguishing face which identifies the face utilizing cascade classifier and for classification and feature selection SVM and PCA is used respectively This proposed system saves time and reduces redundancy when it is being differentiated with the traditional attendance system. 
The Automated System can be employed in registering the participation through a high-resolution computerized camera that perceives and compares the student's face with the faces already stored in the database. Once they are matched, they are given attendance. If it doesn't, then new entries of faces are created. According to [2] portray some imperative viewpoints for face detection which is basic in numerous applications like face recognition, facial expression, face tracking, sexual orientation order, identification framework, record control, and access control, gathering, biometric science and so on. They also emphasized some popular face detection techniques since until we separate the basic highlights (mouth, eyes, nose) from a face we won't be ready to distinguish the person as a unique character. The paper additionally contains an overall discussion on the various phases of any face detection system. Additionally, some other mainstream systems are depicted in short. This method has been made to use in several fields like face recognition, facial feature extraction. One can also use Iris Recognition System. For iris detection and recognition, Students have to face the camera. If the face matches the database, attendance for the student will be given. Various algorithms comprising Grey Scale Conversion, Skin Pixel Detection and many more are used for iris detection. This evacuates the proxy issues and keeps up the attendance in an organized way. But in this process, only one face at a time is detected, which is more time-consuming. Iris detection works for a particular person. The students are asked to come in front of the camera to assist the camera for scanning the iris of the students. The scanned iris is compared with the database containing the already scanned data and after matching attendance is updated. Hence, it lessens the pen and desk workload of the organization.In Fingerprint Recognition system attendance is taken through a portable fingerprint gadget which will contain the fingerprints of all the students. Before and after the class hours, the student needs to give attendance with the help of the configurated device. So that attendance will be given for students who attended the whole lecture. In RFID (Radio Frequency Identification) System, students need to bring their identity card embedded with chip during class hours. During the attendance process, they have to put their id card near the card reader to get their presence recorded. The framework can connect RS232 for recording the participation to the saved database. Some students may misuse other student's id for the attendance if that particular student is not present.

\section{METHODOLOGY}

To begin the process, we need to create a database of students containing their academic information as well as multiple images from different angles and positions. All the images that are captured are stored in a folder named "faces" which contains sub-folders named with student Id. Each sub-folder has multiple images stored for each student. In Fig. I display the architecture of the proposed system. The first step starts with the creation of data by capturing images of every individual student. The second step is the processing step that begins with finding human faces from an image and focusing on by implementing Haar Feature-based Cascade algorithm. Third step is extraction of facial features are called face encodings using face_recognition library then it compares the features encodings with trained data. Finally, by clicking on confirm attendance option attendance is marked for that particular date if face encodings are matched of input image with the trained image. In order to train the system, it requires at least two images of every individual student for better results multiple images are required. In our work, we have used the OpenCV library which has $\mathrm{C}++$, Python and Java interfaces that support Windows, MacOS, and Android. The library supports Computer Vision and Machine Learning algorithms, nearly 2500 optimized algorithms that can be used to detect and recognize faces, track moving objects, identify objects and so on. In coding part ageitgey's face_recognition API for Python is used which is built using dlib's face_recognition algorithms making it easy in face detection and recognition, tracking of objects. The model allows users to perform face recognition from the command line on images stored in a folder. Face encodings- Face encodings are generated for faces found in an image, representing a human face in a 128-dimensional vector. Different images of similar persons are nearer to each other and for dissimilar persons, it is apart. By mapping human to 128-dimensional vector, we can find their Euclidean distance and check whether the distance is small or not. Once Face encodings are generated, it is trained by the KNN classifier with scikit-learn having labels of all known faces. Using face encodings, we can find the nearest neighbour to it at value $\mathrm{k}=1$, and check if matches are thereby fixing a threshold value as 0.6 for distance metric because higher the threshold value more is the chances of misclassifying an unknown student into known one. When predictions are done the classifications are removed if they do not lie within threshold boundary. KNN (K-Nearest Neighbour) algorithm is used because it is a simple machine learning algorithm having straightforward calculations. The algorithm depends on the distance between data points and on that basis, the grouping is done. The algorithm is mostly used in classification as well as regression analysis justifies with the performance when tested on ease in implementations and calculation time parameters. It gives a fair result. In our work, we preferred using the KNN algorithm because it can be trained with the smaller dataset and fetch better results in a minimum time span with minimal hardware requirements. For implementing K- Nearest Neighbour classifier, distance metric function is required, applying between training sample and test sample using "(1)". Let the input samples be $Y_{\sigma}$ having $\mathrm{q}$ features $\left(y_{a 1} \times y_{a 2 \cdots x} y_{a q}\right)$ and the input samples be $m$ where $(a=1,2 \ldots m)$ and number of features be $\mathrm{q}$ where $(b=1,2 \ldots \ldots q)$. Commonly used Euclidean Distance function between samples $r$ and $s$ is defined as

$$
d\left(r_{s} s\right)=\sqrt{\sum_{i-1}^{N}\left(r_{i}-s_{i}\right)^{2}}
$$

Different Distance metric functions can be used or deployed. As in Fig. II, with increasing the value of $k$ the boundary line becomes more and more smoother which will mean lower variance but increase the bias. If the value of $\mathrm{k}$ is smaller, the

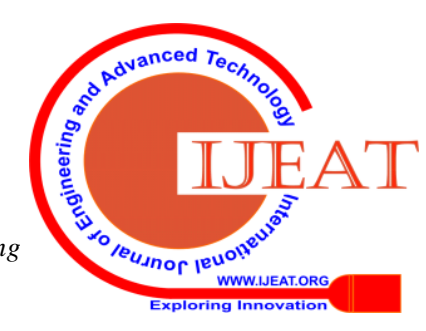



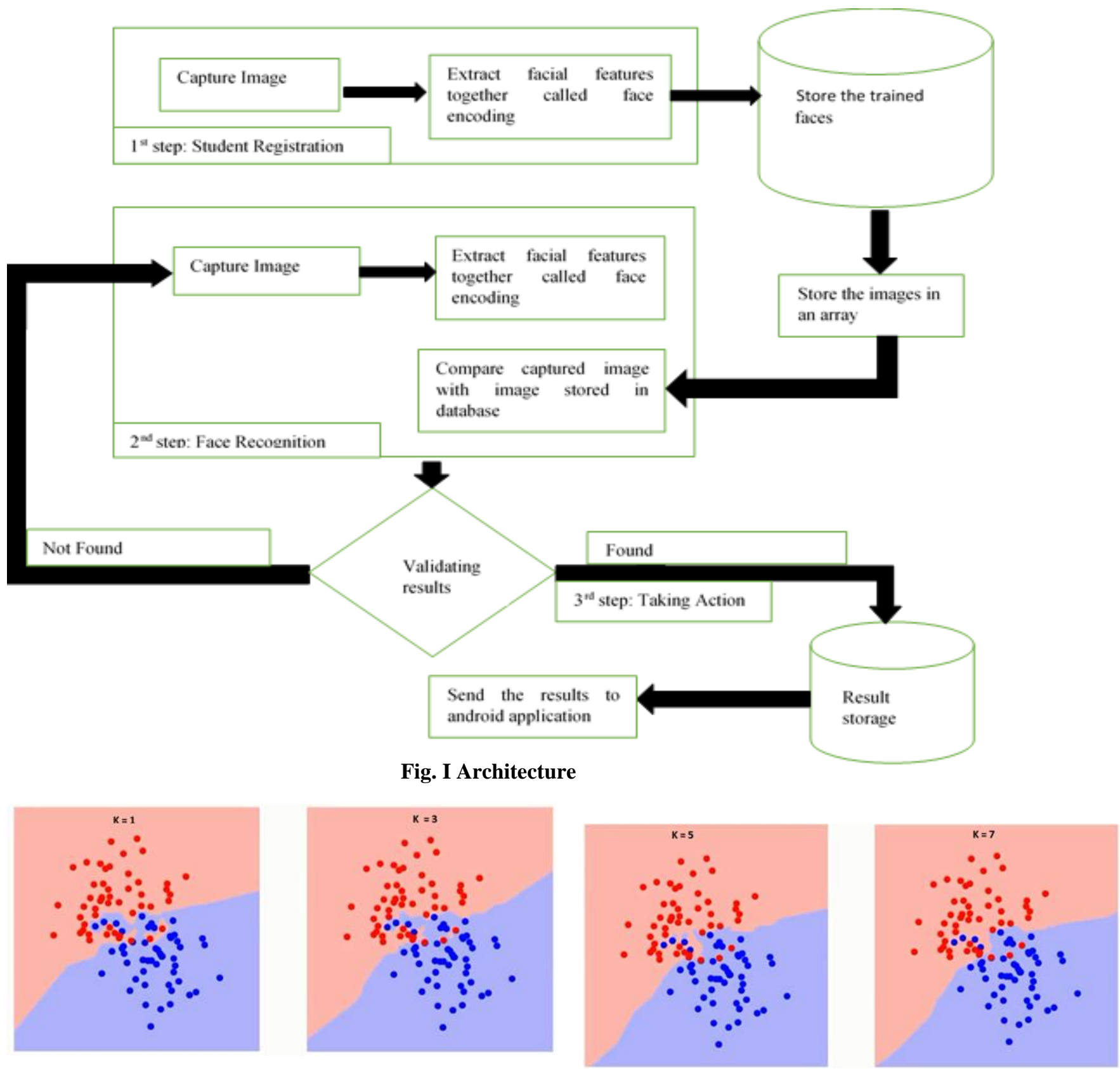

Fig. II Different values of $\mathbf{k}$

final results will include noises and if the value of $\mathrm{k}$ is higher, computation costs are higher. So, if $\mathrm{k}=1$, the error rate for training samples is zero as because the nearest point, i.e., the shortest distance measured can be zero, it will appoint itself as the nearest neighbour but it badly overfits. Hence in most cases, $k=1$ gives accurate results. But an easy way to set the value of $k$ is $k=s q r t(n)$ where $n$ is classes.

When images are captured, it is tested based on the distance of each facial encoding with other images encoding stored in trained dataset. The distance can be found using distance metric formula. Let $\mathrm{P}$ be the known vector and I be the unknown vector. Using distance function, $\mathrm{L}^{2}$ is the distance between $\mathrm{P}$ trained face in data set and the unknown face as an input is calculated. Distance is calculated $\mathrm{i}$ times where is the number of students present in database, according to KNN algorithm lowest the distance between them more is the probability of being a same object as shown in Fig. III.

Here we always use $k=1$ for face recognition, as $P_{i}$ represents the student's face and main motive is discover unique identity of I. As in Fig. III there is least distance $L$ between I and $\mathrm{P}_{1}$, so it can be predicted that I belongs to $\mathrm{P}_{1}$ object. If we put as $\mathrm{k}=2$, the identity of $\mathrm{I}$ will be for $\mathrm{P}_{1}$ as well as $\mathrm{P}_{2}$, which is not required in face recognition. Further, if the distance $L^{2}$ is the lowest value, then it is called The Confidence.

Once student's face is captured by mobile camera for marking attendance, clicking on find student option checks whether the new image matches from trained images, if it is matched the information is displayed in the screen itself and if it is not matched no information will be displayed which means this student is 


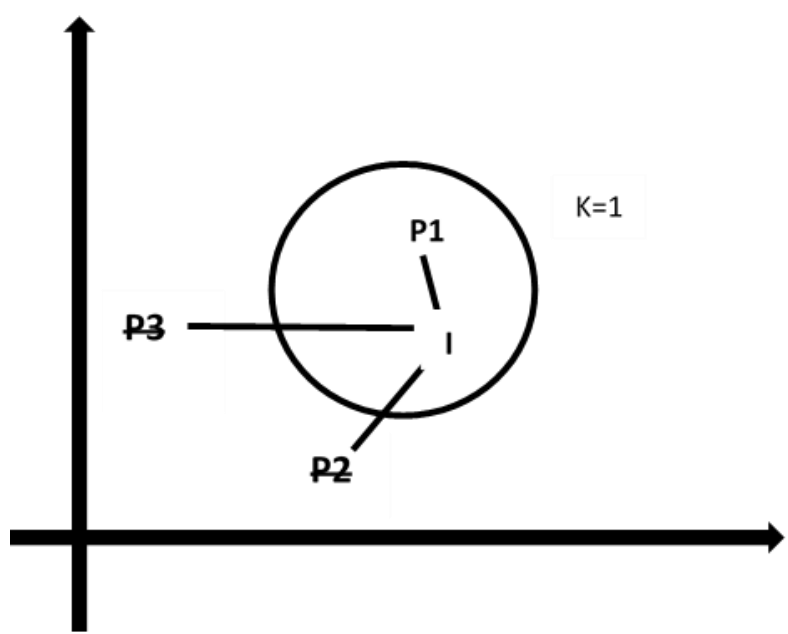

Fig. III KNN Algorithm with

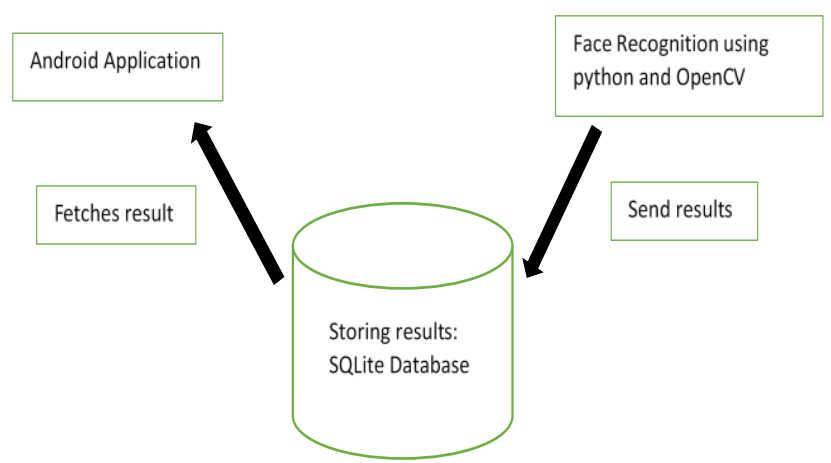

Fig. IV connection between face recognition and the system

new or does not belong to a particular class. Finally, clicking on confirm attendance option attendance is given to that particular student and results are sent to the database from the android system as Fig. IV shows the connection of the database with the android application. In this project, we have used SQLite which is a SQL database, and android comes with built-in SQLite database implementation.

\section{RESULTS AND DISCUSSIONS}

The main ideology of the model is to reduce the data redundancy by making the system more efficient and handier using an android based Mobile Application, for real-time Face-Recognition Modern Attendance System. Android app is installed with apk files only in permitted mobile phones. Beginning with, a user has to feed login credentials to get access to the framework.

Using the app, Student details are added as shown in Fig. VII including images, name, Registration number, academic year and so on which get stored in the database. After the addition of images, it gets trained with the KNN model. In our work we trained with 5 images of each student as shown in Fig. V.

For face recognition, 2 python codes were written, one for training images with KNN classifier, and others for running the server and the recognizing student's face which is the input.

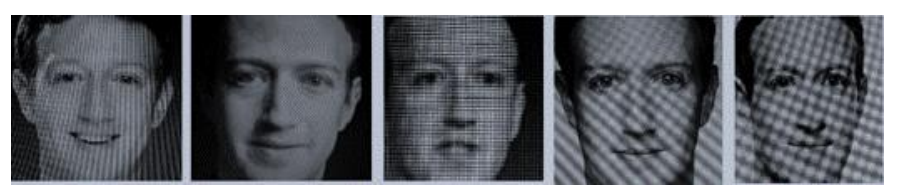

Fig. V trained images of a student

Retrieval Number: D7710049420/2020@BEIESP DOI: 10.35940/ijeat.D7710.049420

Journal Website: www.ijeat.org
To give attendance, click on Take Attendance button, and the new frame is displayed as shown in Fig. VI, that will capture new images of the individuals and then click on find student to check whether the particular student belongs to that class. The new image is compared with existing images stored in the database and based on similarities present in their features it shows the belongingness.

Anytime one can check the attendance and information's for a particular student from the app itself and date too for which he/she got attendance.

Recognition Results-The student details are displayed if their data exists in the database as shown in Fig. VIII and if details are not popped up, null will be displayed airing the failure in recognition if the student is not added as shown in Fig. IX. After verification clicking on confirm, the attendance button will mark attendance for a particular student. If the information is not displayed, then again add student procedure will be repeated and then attendance can be marked. Here the proxy issues, as well as redundancy, are minimized.

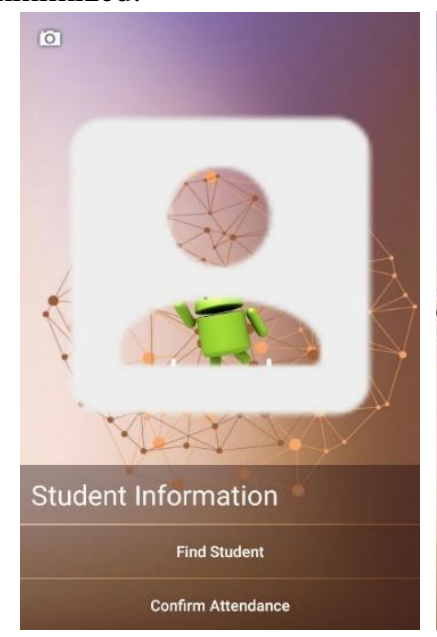

Fig. VI Take Attendance

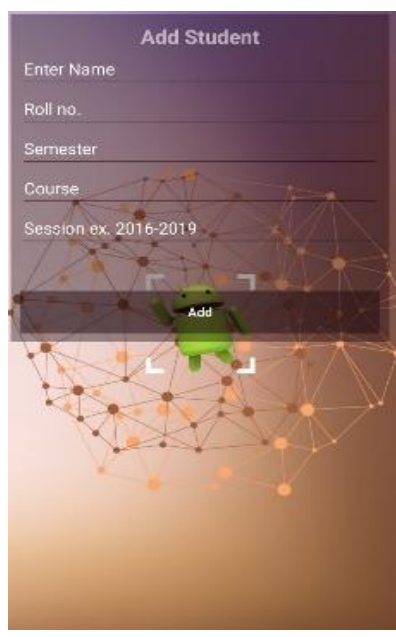

Fig. VII Add Student
Moreover, the accuracy of the result is $94 \%$ when $\mathrm{k}=1$, if the value of $\mathrm{k}$ is increased the accuracy slightly decreases as higher the value of $\mathrm{k}$ more will be the search points in the neighborhood. But if the LBPH algorithm is implemented in this system the accuracy is $45 \%$ which is relatively low when compared with the present algorithm used in this system. When face matches when the threshold value is 0.6 and 0.4 , the result looks like as shown in Fig. X. The confidence score is calculated by building test sets and evaluating results upon it.

\section{SYSTEM LIMITATION}

The present system has limitations using KNN Algorithm. As KNN is sensitive to noise present in the dataset, missing values, as well as outliers, are removed manually because it can strongly effect on regression analysis. The system does not work well with a huge set of images as the KNN classifier is a slow learner.

Moreover, Accuracy also varies when the image is blur or dark. Depending upon brightness and pixel quality of an image accuracy differs. If the image blurs the accuracy decreases.

Published By:

Blue Eyes Intelligence Engineering

\& Sciences Publication

(C) Copyright: All rights reserved.

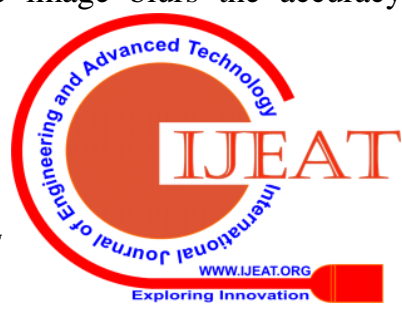




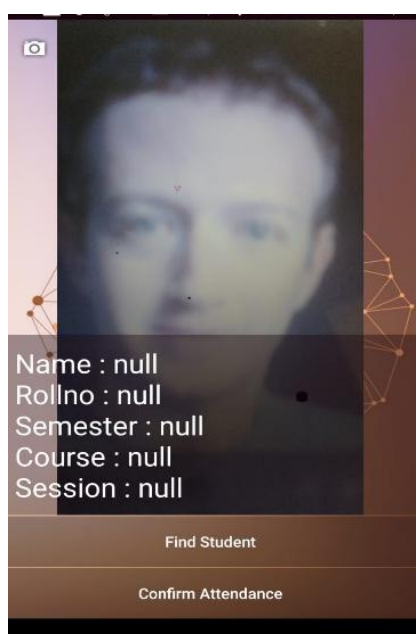

Fig. VIII Face is not Known

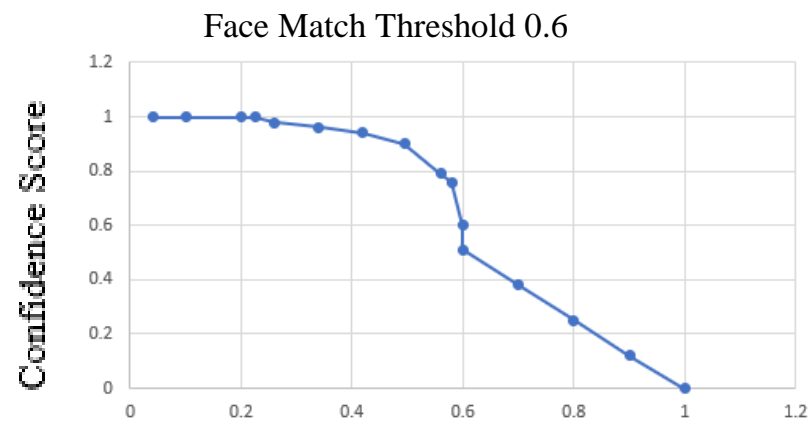

Face Distance

Face Match Threshold 0.4

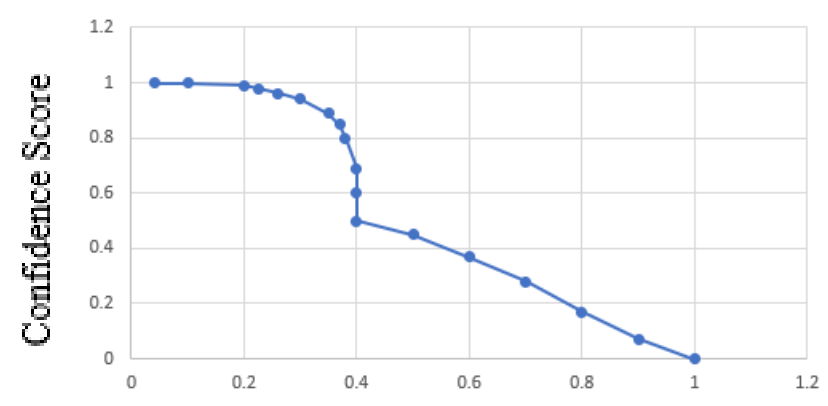

Face Distance

Fig. $X$ (a) Threshold=0.6 (b) Threshold $=0.4$

\section{CONCLUSIONS}

The main motive of this research paper is to reduce pen and paperwork and make this system one step towards automation and artificial intelligence. Reliable detection and recognition of facial expressions from still images in the unconstrained real-world situations has many potential applications. The conventional method of taking attendance has several disadvantages system like time taking and requiring more human efforts, duplicate data entry, errors in attendance entries, proper process not available to track and manage student attendance status in real time, no proper security and confidentiality with role-based permission to users etc. This proposed system reduces the anomalies that occur during the attendance process. The system is easy to use as nowadays everyone uses the android application. As no complex hardware tools and cameras are required. The system is cost-effective too, no extra expenses are required. The system helps in enhancing the speed that leads to achieving the good-accuracy in giving attendance to students in the classroom. Hence, attendance is marked with a single click on the screen and the record of the attendance will also be stored in the database for further assessment. The app will have a student's previous records and will update every day after the attendance is taken. It also has an increased security and confidentiality. This attendance system helps in enhancing the accuracy and speed that helps in achieving the high-accuracy in ongoing participation to address the issue for programmed classroom assessment.

Further improvements can be made using neural networks and detecting multiple faces from a single picture at a time.

\section{REFERENCES}

1. Nandhini R, Duraimurugan N, S.P.Chokkalingam, "Face Recognition Based Attendance System", (IJEAT) ISSN: 2249 - 8958, Vol.-8, Issue3S, Feb2019.

2. Mrs. Sunita Roy and Mr. Susanta Podder "Face detection and its applications", IJREAT, Vol. 1, Issue 2, April-May, 2013."

3. O. A. R. Salim, R. F. Olanrewaju and W. A. Balogun, "Class Attendance Management System Using Face Recognition," 2018 7th (ICCCE), Kuala Lumpur, 2018, pp. 93-98.

4. M. H. Yang, D. J. Kriegman, and N. Ahuja, "Detecting face in images: a survey," IEEE Trans. Patter Analysis and Machine Intelligence, vol. 24, pp. 34-58, 2002.

5. Manjunath, B., Chellappa, R. \& von der Malsburg, C. (1992), „A Feature Based Approach to Face Recognition “e, IT Department, MVGR College of Engineering, Vizianagaram,IEEE Conference Proceedings on Computer Vision and Pattern Recognition pp. 373-378.

6. A. Vardhan K, "Portable Attendance Monitoring system using face Recognition", (ICCIIoT) 2018.

7. Shireesha Chintalapati, M.V. Raghunadh, "Automated Attendance Management System Based On Face Recognition Algorithms", IEEE International Conference on Computational Intelligence and Computing Research, 2013.

8. H.A. Rowley ; S. Baluja ; T. Kanade, "Rotation invariant neural network-based face detection" Proceedings. 1998 IEEE Computer Society Conference on Computer Vision and Pattern Recognition (Cat. No.98CB36231).

9. Rosebrock, Adrian. "k-NN Classifier for Image Classification." PyImageSearch, 8 Aug. 2016, www.pyimagesearch.com/2016/08/08/k-nn-classifier-for-image-classi fication/.

10. Peterson, Leif E. "K-Nearest Neighbor." Scholarpedia, 21 Feb. 2009 www.scholarpedia.org/article/K-nearest neighbor.

11. Imran. "How to Choose the Value of $\mathrm{K}$ in Knn Algorithm." Data Science, Analytics and Big Data Discussions, 2 Aug. 2015, discuss.analyticsvidhya.com/t/how-to-choose-the-value-of-k-in-knn-a lgorithm/2606

12. R. K. Mishra1, Vijay Kr Bhati2, Jagdish Nishad3, Richa Mehra "Real Time Face Recognition Based Attendance Monitoring System" (IJRASET) May 2018.

13. Sakshi Patel, Prateek Kumar, Shelesh Garg , Ravi Kumar, "Face Recognition based smart attendance system using IOT" IJCSE Vol.-6, Issue-5, May 2018 E-ISSN: 2347-2693.

14. Ageitgey, Adam. “Ageitgey/face_recognition.” GitHub, 9 Mar. 2018 github.com/ageitgey/face_recognition/blob/master/examples/face_rec ognition_knn.py.

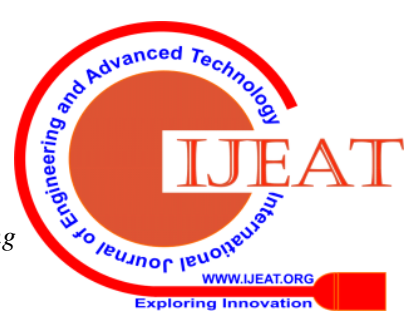




\section{AUTHORS PROFILE}

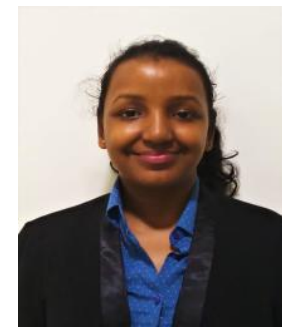

Tanya Sinha, a final year student currently pursuing B-tech degree in Computer Science and Engineering with segregate $90.3 \%$ at SRMIST KTR Campus, Tamil Nadu. The interests include Machine Learning, Internet Of Things and Big Data. I have also done research in comparative study of different Machine Learning Algorithms. Also, worked in club Gen-y as a member of machine Learning Domain.

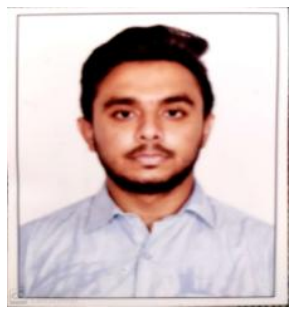

Abhisekh Ghosh currently pursuing final year of B.Tech in Computer Science Engineering at SRMIST, Tamil Nadu. He will be completing his undergraduate degree by june 2020 and has keen interest in Machine learning and artificial intelligence. He has done works on several fields of embedded systems in his academic career.

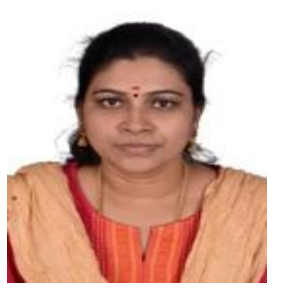

I am currently working as Assistant Professor in SRM Institute of Science and Technology. The research interests of the authors include: machine learning, text mining, social network analysis and the recommender system. I graduated with a Bachelor degree in Computer Science and Engineering from University of Madras. Further, graduated with a Master's degree and $\mathrm{PhD}$ from College Of Engineering, Guindy, Anna University. I have published around 10 papers in various Conferences, National and International Journals.

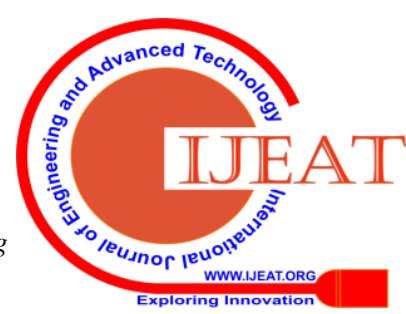

